\title{
Pedicled anterolateral thigh flap for contralateral groin composite defect
}

\author{
S. P. Bharath, G. Madhusudan, Suraj Manjunath ${ }^{1}$ \\ Department of Plastic, Reconstructive Surgery and Burns and 'Department of Surgical Oncology, St. John's Medical College \\ Hospital, Sarjapur Road, John Nagar, Bangalore, India
}

Address for correspondence: Dr. S.P. Bharath, Department of Plastic, Reconstructive Surgery and Burns, St. John's Medical College Hospital, Sarjapur Road, John Nagar, Bangalore, India. E-mail: drbharathsp@yahoo.co.in

\section{ABSTRACT}

Pedicled anterolateral thigh flap has been well described for ipsilateral groin defects. Its versatility depends on the intact femoral vessels. When the external iliac and the femoral vessels are absent, especially secondary to wide surgical tumour ablations in the groin region, ipsilateral ALT flap is not an option. Free flaps also are difficult because of lack of recipient vessels. We report a case of composite groin defect following wide resection of recurrent liposarcoma along with encased vessels which was covered with a pedicled anterolateral thigh flap from the opposite thigh. The technique of lengthening the vascular pedicle and medializing the pedicle, to effectively increase its reach to the contralateral anterior superior iliac spine without vascular compromise, is described.

\section{KEY WORDS}

Pedicled flap; anterolateral thigh myocutaneous flap; contralateral groin defect

\section{INTRODUCTION}

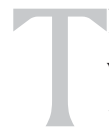

he pedicled anterolateral thigh flap has been well described for ipsilateral groin defects as a proximally based flap. In our case, we have used the ALT flap to cover a contralateral groin composite defect, demonstrating its reachability to the opposite anterior superior iliac spine by medializing the pedicle under the rectus femoris muscle.

\section{CASE REPORT}

A 65-year-old female presented with recurrent liposarcoma of left groin and upper thigh of six months duration, following excision and radiotherapy four years ago. This $8 \times 8 \mathrm{cms}$ tumour was adherent to the previous surgical scar. The femoral pulse was not palpable due to encasement of the vessels as confirmed by MRI. Radical en-mass resection of the lesion with adequate margins included skin with previous surgical scar, whole of left inguinal ligament, partial left superior pubic ramus and retroperitoneal clearance with removal of external iliac - femoral vessels up to the upper thigh. The continuity of vessels to left lower limb was restored by PTFE grafts and a polypropylene mesh was used to reinforce the peritoneal defect at the inguinal region.

This defect of $15 \times 22 \mathrm{cms}$ with exposed PTFE grafts and the mesh [Figure 1] posed a new reconstructive challenge due to non availability of ipsilateral pedicled flaps as a long length of the external iliac and femoral vessels including the profunda were resected. Free flap transfer due to want of recipient vessels was not an option. Local random flaps were impractical due to the large size of the defect and previous irradiation. 
The available donor sites were the opposite anterior abdominal wall and thigh. In view of extensive dissection and undermining of the anterior abdominal wall, we chose a flap from the contralateral thigh.

\section{Pedicled contralateral ALT transfer}

The desired skin paddle was marked $(22 \times 15 \mathrm{cms})$ around a perforator identified by hand held Doppler. After elevating the skin paddle three musculocutaneous perforators through the vastus lateralis were identified without any septocutaneous perforators. Most of vastus except the lateral third was harvested with the overlying skin paddle. The length of descending branch of lateral circumflex femoral vessels obtained was $12 \mathrm{cms}$ after ligating the branch to rectus femoris. The transverse branch was also ligated and further proximal dissection up to its origin from profunda gave an additional $3 \mathrm{cms}$ to the pedicle length. The flap was tunnelled under the rectus femoris which provided unfolding of the 15 cms long pedicle for easier swing to the opposite groin

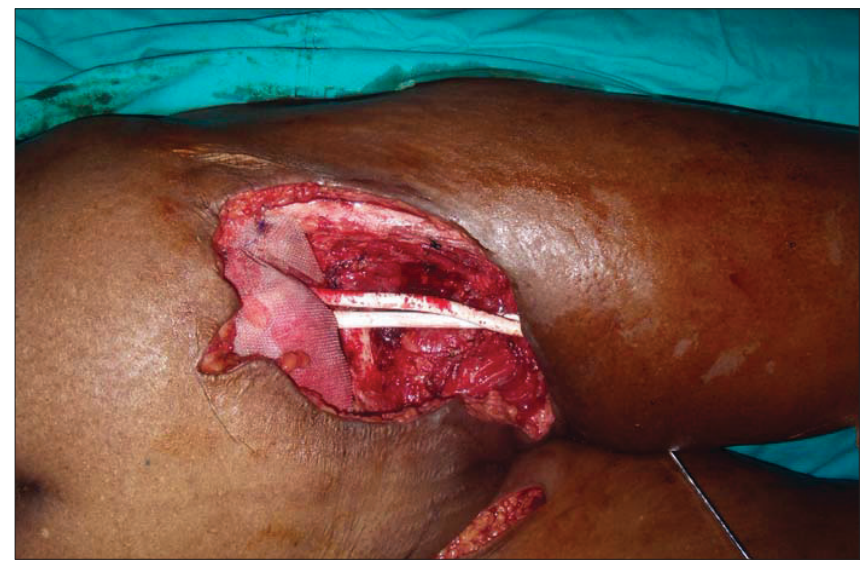

Figure 1: Left groin composite defect

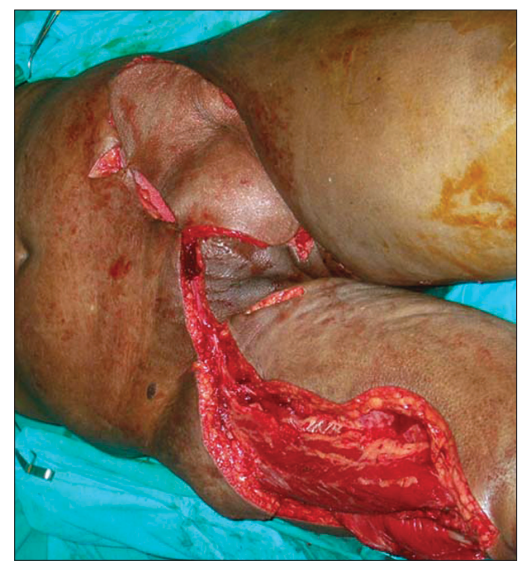

Figure 3: ALT flap covering the defect after extended dissection and tunnelling under rectus femoris medialising the pedicle
[Figures 2-4]. The pedicle was buried subcutaneously in the supra pubic region. The vastus muscle provided good bulk and the tendinous portion reached the opposite anterior superior iliac spine. The flap covered the composite defect comfortably without any stretch on the pedicle. The donor site was skin grafted and all other incisions closed primarily.

The flap and the donor site graft healed well. There was lymphorrhea from under the flap which was managed by a drain for three weeks.

\section{DISCUSSION}

Complex groin defects after cancer ablation can be large and difficult to resurface. ${ }^{[1]}$ The most common reconstructive donors come from the ipsilateral thigh as muscle (rectus femoris, sartorius and tensor fascia lata) or myocutaneous extensions or skin flaps like ALT flap (septocutaneous or myocutaneous) or local random

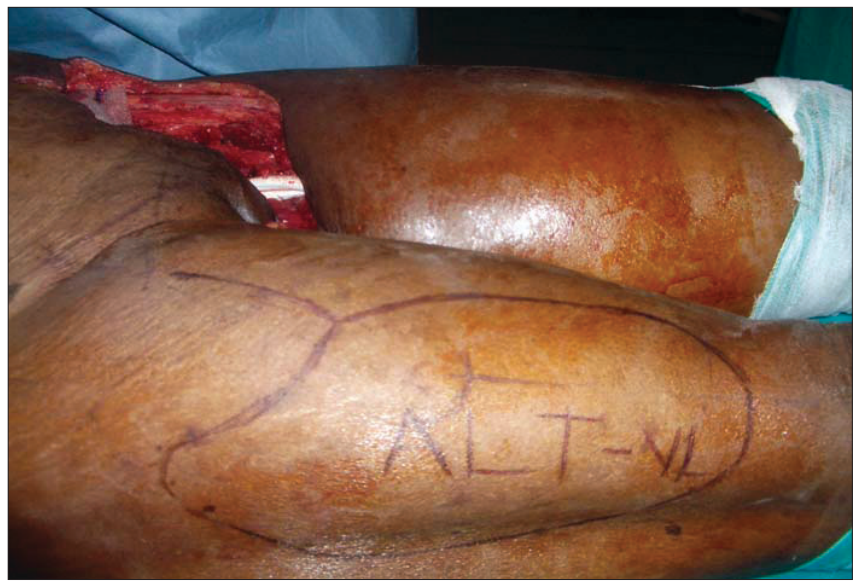

Figure 2: Contralateral right anterolateral thigh flap marked

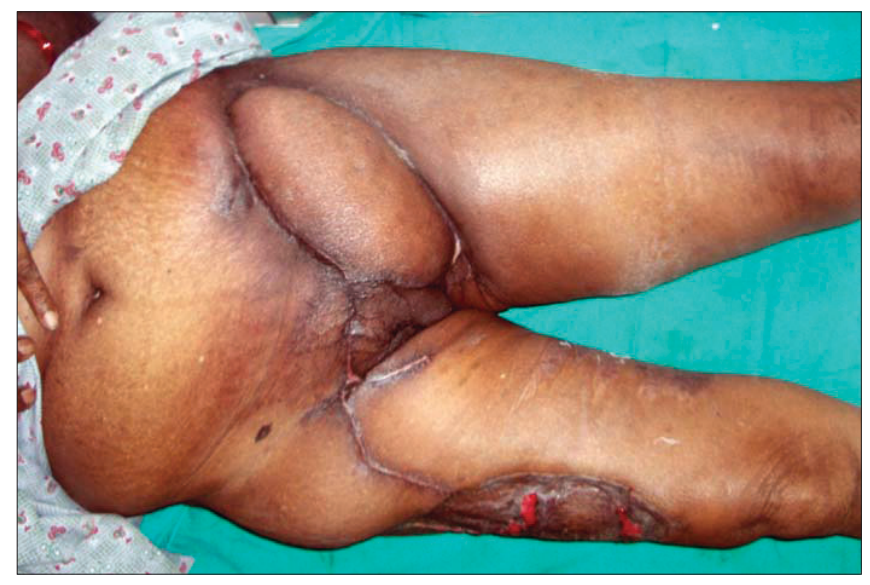

Figure 4: Healed ALT flap and the donor thigh at 3 weeks 
flaps. ${ }^{[2]}$ Since all these flaps are based on the proximal femoral artery branches, its integrity is of prime importance in the success of the flap. In our case the resection of the external iliac and femoral vessels up to the proximal third of the thigh posed a difficult reconstructive task as the ipsilateral thigh and anterior abdominal wall could not be used as a donor site and the free flap was impractical due to the want of recipient vessels in the vicinity. The local random skin flap transposition was not enough to cover the huge composite defect as the latter needed both good filler and a cover. We were left with options from the contralateral abdominal wall and thigh. We chose thigh over abdominal wall as the latter would have caused difficulty in donor site closure in view of wide undermining of the ipsilateral side.

Since the first description of ALT flap by Song et $a l,{ }^{[3]}$ as a free flap, it has been described for ipsilateral groin, ${ }^{[4]}$ trochanteric, ischial and genital defects as a proximally based flap and for knee defects as distally based flap. ${ }^{[5]}$ In our case, we used the ALT flap pedicled from the contralateral thigh, demonstrating its extended arc of rotation to the opposite anterior superior iliac spine without compromising the vascularity of the flap. The length of the robust vascular pedicle of descending branch of lateral circumflex femoral artery was increased to $15 \mathrm{cms}$ (Lee et al. ${ }^{[6]}$ reported that it can range from $8.5 \mathrm{~cm}$ to $14 \mathrm{~cm}$ ) by dissecting up to its origin from profunda femoris as described above. Though there is mention of extended dissection under the rectus in achieving long pedicle in free flaps, ${ }^{[7]}$ there is no report of such a proximally pivoted pedicle with an effective gain in length of $15 \mathrm{cms}$. Vascular compromise of the rectus femoris due to such extensive skeletonisation of the pedicle, though rare has to be borne in mind. The arc of rotation up to 150 degrees in reaching the opposite groin was possible only after medializing the flap by tunnelling under rectus femoris. This shifted the pivot point of the pedicle medial to the rectus and helped in the swing of the flap to the opposite groin. In a series of 17 pedicled ALT flaps, Ahmed et al. ${ }^{[8]}$ describe tunnelling of the flap between the rectus and the fascial septa for ipsilateral inguinal defects. In our case, complete medial unfolding of the flap pedicle on the trunk of the profunda vessels was done to achieve the reach. We buried the pedicle in a lay open incision at the suprapubic area which may be a drawback of the procedure as it may not be acceptable in young patients.

\section{CONCLUSION}

The pedicled ALT flap can be reliably and safely used to cover complex defects of the contralateral groin aided by the extended dissection and medialisation of the pedicle by tunnelling it under the rectus femoris muscle increasing the arc of rotation. This technique can thus be extended to cover the contralateral inguinal, iliac and abdominal wall defects in selected cases where the ipsilateral options are limited.

\section{REFERENCES}

1. Evriviades D, Raurell A, Perks AG. Pedicled anterolateral thigh flap for reconstruction after radical groin dissection. Urology 2007;70:996-9.

2. Mathes SJ, Nahai F. A systematic approach to flap selection: Groin. In: Mathes SJ, Nahai F, editors. Clinical applications for muscle and musculocutaneous flaps. The CV Mosby Company; 1982. p. 388-425.

3. Song TG, Chen GZ, Song YL. The free thigh flap: A new free flap concept based on the septocutaneous artery. Br J Plast Surg 1984;37:149-59.

4. Tiguemounine J, Picard A, Fassio E, Goga D, Ballon G. Uterine liposarcoma invading abdominal wall and inguinal region: Immediate reconstruction using a pedicled anterolateral thigh flap. Ann Chir Plast Esthet 2003;48:180-6.

5. Gravvanis AI, Tsoutsos DA, Karakitsos D, Panayotou P, Iconomou $\mathrm{T}$, Zografos $\mathrm{G}$, et al. Application of the pedicled anterolateral thigh flap to defects from the pelvis to the knee. Microsurgery 2006;26:432-8.

6. Lee JT, Cheng LF, Lin CM, Wang CH, Huang CC, Chien SH. A new technique of transferring island pedicled anterolateral thigh and vastus lateralis myocutaneous flaps for reconstruction of recurrent ischial pressure sores. J Plast Reconstr Aesthet Surg 2007;60:1060-6.

7. Spyriounis PK. The extended approach to the vascular pedicle of the anterolateral thigh perforator flap: Anatomical and clinical study. Plast Reconstr Surg 2006;117:997-1001.

8. Ahmad QG, Reddy M, Shetty KP, Prasad R, Hosi JS, Bhathena M. Groin reconstruction by anterolateral thigh flap: A review of 16 cases. Indian J Plast Surg 2004;37:34-9.

Source of Support: Nil, Conflict of Interest: None declared. 